\title{
BMJ Open von Willebrand factor as a biomarker of clinically significant portal hypertension and severe portal hypertension: a systematic review and meta-analysis
}

\author{
Ziyuan Zou, ${ }^{1,2}$ Xinwen Yan, ${ }^{\oplus 2}$ Cheng Li, ${ }^{3}$ Xiaofeng Li, ${ }^{4}$ Xiaofen $\mathrm{Ma},{ }^{5}$ \\ Chunqing Zhang, ${ }^{6}$ Shenghong Ju, ${ }^{7}$ Junzhang Tian, ${ }^{5}$ Xiaolong Qi ${ }^{1}$
}

To cite: Zou Z, Yan X, Li C, et al. von Willebrand factor as a biomarker of clinically significant portal hypertension and severe portal hypertension: a systematic review and meta-analysis. BMJ Open 2019;9:e025656. doi:10.1136/ bmjopen-2018-025656

- Prepublication history and additional material for this paper are available online. To view these files, please visit the journal online (http://dx.doi org/10.1136/bmjopen-2018025656).

$\mathrm{ZZ}, \mathrm{XY}$ and $\mathrm{CL}$ contributed equally.

Received 27 July 2018 Revised 28 June 2019 Accepted 03 July 2019

Check for updates

(C) Author(s) (or their employer(s)) 2019. Re-use permitted under CC BY-NC. No commercial re-use. See rights and permissions. Published by BMJ.

For numbered affiliations see end of article.

Correspondence to

Prof Xiaolong Qi;

qixiaolong@vip.163.com

\section{ABSTRACT}

Objective This meta-analysis was performed to investigate the correlation between von Willebrand factor (vWF) antigen and hepatic venous pressure gradient (HVPG) and to evaluate the diagnostic performance of vWF to detect clinically significant portal hypertension (CSPH) and severe portal hypertension (SPH).

Design Systematic review and meta-analysis. Methods MEDLINE, EMBASE, Web of Science and the Cochrane Library were screened up to 5 April 2018. Studies related to the diagnostic performance of vWF to detect CSPH and/or SPH with HVPG as the reference standard were included. Study quality was assessed by using the Quality Assessment of Diagnostic Accuracy Studies scale. Two authors independently used a standardised form to extract data.

Outcomes The primary outcome was the correlation coefficient between vWF and HVPG. The secondary outcome was the diagnostic performance of VWF to detect CSPH or SPH.

Results A total of six articles involving 994 patients were included in this study. Five of the included articles were used to stratify the results for the correlation coefficient, three for the diagnostic performance of CSPH and two for SPH. The pooled correlation coefficient based on the random effects model was 0.54 (95\% $\mathrm{Cl} 0.35$ to 0.69$)$, thus suggesting a moderate correlation between vWF and HVPG. The pooled sensitivity, specificity and area under the curve of vWF for CSPH detection were $82 \%(95 \% \mathrm{Cl} 78$ to 86$), 76 \%$ (95\% Cl 68 to 83$)$ and $0.87(95 \% \mathrm{Cl} 0.80$ to $0.94)$, respectively. Regarding the ability of vWF to detect $\mathrm{SPH}$, the pooled sensitivity and specificity were $86 \%(95 \%$ Cl 80 to 90) and 75\% (95\% Cl 66 to 83), respectively.

These results supported the satisfactory diagnostic performance of vWF for CSPH and SPH detection. Conclusions vWF, as a novel biomarker, has a moderate correlation with HVPG and shows a satisfactory performance for the diagnosis of CSPH and SPH in patients with cirrhosis.

\section{INTRODUCTION}

Portal hypertension $^{1}(\mathrm{PH})$ accounts for the major complications of liver cirrhosis, including decompensation, ascites and variceal haemorrhage. ${ }^{23}$ When decompensation

\section{Strengths and limitations of this study}

- Our research focused on more detailed categories, which were defined as clinically significant portal hypertension (hepatic venous pressure gradient (HVPG) $\geq 10 \mathrm{~mm} \mathrm{Hg}$ ) and severe portal hypertension (HVPG $\geq 12 \mathrm{~mm} \mathrm{Hg}$ ), and evaluated diagnostic accuracy.

- We calculated the diagnostic accuracy of von Willebrand factor (vWF) and investigated the correlation between VWF and HVPG, the gold standard of portal hypertension, to demonstrate the comprehensive diagnostic efficiency of this non-invasive biomarker

- This meta-analysis involved a relatively small number of studies (ie, six studies) of which two were retrospective with low-level evidence, thus giving rise to restrictions in the test methods and cut-offs.

- The eligible population of the included studies differed slightly in aetiology.

- The between-study source of heterogeneity was not found in this meta-analysis.

occurs, the expected median survival is approximately 2 years. ${ }^{4} 5$ Thus, for the management of patients with cirrhosis, the early diagnosis of $\mathrm{PH}$ is essential. Previous investigations $^{1}{ }^{2}$ demonstrated that with adequate treatment after early diagnosis, the mortality of PH-related complications can be significantly reduced.

Recent guidelines recommend measuring the hepatic venous pressure gradient $(\mathrm{HVPG})^{6}$ for the diagnosis of $\mathrm{PH}^{3}{ }^{3}$ A higher risk of varices, other PH-related complications and liver-related death has been found to be associated with clinically significant portal hypertension $(\mathrm{CSPH})$ (HVPG $\geq 10 \mathrm{~mm}$ $\mathrm{Hg}){ }^{7}$ Additionally, a higher risk of bleeding from varices is related to severe portal hypertension (SPH) (HVPG $\geq 12 \mathrm{~mm} \mathrm{Hg}$ ). However, HVPG is an invasive clinical index that can only be conducted in specialised 
centres. Thus, non-invasive biomarkers would be of great benefit for the diagnosis and prognosis of patients. ${ }^{8}$ The significance of non-invasive markers that have entered the clinical routine provides evidence of their satisfactory specificity and sensitivity. ${ }^{8}$ Furthermore, transient elastography (TE) based on FibroScan was recently described as a non-invasive tool for the diagnosis of $\mathrm{PH}$ in patients with liver disease. ${ }^{9}$ However, the availability and costs of TE restrict its use in smaller hospitals. ${ }^{9}$

In cirrhotic livers, increased intrahepatic vascular resistance is considered to be associated with endothelial dysfunction. ${ }^{7}$ The von Willebrand factor (vWF) antigen, a multimeric adhesive protein, is released by activated endothelial cells and is responsible for the endothelium interaction at sites of vascular injury and in platelets. Importantly, vWF levels are commonly elevated in patients with cirrhosis ${ }^{10}$ and are considered to be a marker of endothelial dysfunction, ${ }^{11}$ in which increased intrahepatic resistance and deteriorated $\mathrm{PH}$ occur. The procoagulant state has been shown to potentially predict hepatic decompensation in patients with cirrhosis. ${ }^{12}$ The fluctuation of vWF values, which can be expressed as a ratio, gives an index of the procoagulant state ${ }^{12}$ which may independently exhibit intrahepatic vascular resistance. Thus, the potential association between vWF levels and procoagulant imbalance might further contribute to its predictive value of complications and mortality in patients with cirrhosis. ${ }^{12} 13$

Although several studies have evaluated the diagnostic performance of vWF to detect CSPH and SPH, some of them were retrospective studies with low-level evidence, and there was an evident lack of consideration of the correlation between vWF and HVPG. Thus, generating a more evidence-based systematic summary for the standardised management of patients with liver cirrhosis would be beneficial. Therefore, we performed this meta-analysis to determine the correlation between $\mathrm{vWF}$ and HVPG and to evaluate the diagnostic accuracy of vWF to detect CSPH and SPH in patients with liver cirrhosis.

\section{MATERIALS AND METHODS}

\section{Literature search strategy}

This meta-analysis was performed according to the recently published recommendations and checklist of the Preferred Reporting Items for Systematic Reviews and Meta-analyses (PRISMA) statement. ${ }^{14}$

A comprehensive electronic literature search was performed to identify original publications with relevant topics from MEDLINE, Web of Science, EMBASE and the Cochrane Library regarding the correlation between vWF and HVPG measurement and the diagnostic performance and its accuracy in diagnosing CSPH and SPH among patients with liver cirrhosis. The following key terms were used: ('vWF' OR 'von Willebrand Factor' OR 'von Willebrand Protein') AND ('cirrhosis' AND 'portal hypertension'). 'Liver cirrhosis' OR 'portal hypertension' was designed for identifying the patient group, while the 'vWF' OR 'von Willebrand factor' AND 'hepatic vein pressure gradient' were designed for the diagnostic index and its contrast. The full search strategy and keywords used in this research are shown in online supplementary appendix methods. A search in the bibliographies of the included articles was also conducted. There was no restriction on the beginning date of the literature search. We searched the literature published before 5 April 2018. All searches were restricted to 'English' and 'humans'. Additionally, the reference lists and cited articles of each study were manually checked to prevent duplication.

\section{Inclusion and exclusion criteria}

The inclusion criteria were as follows: (1) study design: clinical trial or observational study; (2) population: patients with liver cirrhosis; (3) diagnostic measurement: vWF as the index test for diagnosis, while HVPG was a reference standard for comparison; (4) reference standard: CSPH should be defined as HVPG $\geq 10 \mathrm{~mm} \mathrm{Hg}$, and standard SPH should be defined as HVPG $\geq 12 \mathrm{~mm} \mathrm{Hg}$; (5) presence of sufficient data to obtain $2 \times 2$ contingency tables (ie, true positive, false positive, true negative and false negative) or the correlation coefficient between $\mathrm{vWF}$ and HVPG measurement; (6) full texts were accessible; and (7) trials reported in English.

Studies that met the following criteria were excluded: (1) study design: duplicate publication, literature review, systematic review, case report or case series; (2) experimental model: animal or cell; (3) outcomes were not available even after corresponding with the authors; (4) the study outcome was out of the field of interest of this meta-analysis; (5) studies that used other management techniques apart from HVPG as a reference standard; and (6) studies that were not in compliance with the inclusion criteria.

\section{Study selection and quality assessment}

All studies were fully reviewed by two reviewers (XY and $\mathrm{ZZ)}$ according to the inclusion and exclusion criteria. Each reviewer selected the included studies independently in the review section. Any disagreements between the two reviewers were settled by consensus. If a consensus between the two reviewers could not be reached, a third author (XQ) was deferred to for arbitration and consensus.

The methodological quality was estimated using Review Manager V.5.3 according to the Quality Assessment of Diagnostic Accuracy Studies-2 (QUADAS-2) criteria $^{15}$ which has been considerably improved compared with QUADAS $^{16}$ in terms of assessing the quality of included studies. By using QUADAS-2, four domains, including patient selection, index test, reference standard, and flow and timing, are evaluated to identify the risk of bias, and the first three domains are also assessed in terms of concerns regarding applicability. ${ }^{15}$

Disagreements were resolved under discussion between the two review authors (XY and $\mathrm{ZZ}$ ), with a third author as the final referee $(\mathrm{XQ})$. 


\section{Data extraction}

The following data were extracted to standardise data forms from the selected studies: publication year; study design; eligible population(cause of liver cirrhosis); Child-Pugh score; assessment of PH; test methods; median follow-up; outcome of interest; the statistical method and results for investigating the correlation coefficient between vWF and HVPG; a $2 \times 2$ table composed of the value of true positive, false positive, true negative and false negative in which the data were used to calculate the pooled sensitivity, specificity, positive likelihood ratio (PLR), negative likelihood ratio (NLR) and diagnostic OR (DOR). When the HVPG $\geq 10 \mathrm{~mm} \mathrm{Hg}$, PH was defined as $\mathrm{CSPH},{ }^{7}$ whereas HVPG $\geq 12 \mathrm{~mm} \mathrm{Hg}$ was diagnosed as SPH. ${ }^{11}$

\section{Data analysis}

Using Comprehensive Meta-Analysis (V.3, www.meta-analysis.com), MetaDiSC (V.1.4, www.hrc.es/investigacion/ metadisc.html) and $\mathrm{R}$ (V.3.1.0, www.r-project.org), we performed a meta-analysis to calculate (1) the pooled sensitivity, specificity, PLR, NLR and DOR for vWF as a marker of $\mathrm{CSPH}$ and SPH; (2) the summary receiver operating characteristic (SROC) curve and pooled area under the receiver operating characteristic curve (AUC) for vWF as a marker of CSPH; and (3) the pooled correlation coefficient between vWF and HVPG. All values were reported as point estimates with $95 \%$ CIs in parentheses.
Funnel plots were drawn to assess the possibility of publication bias.

Heterogeneity was evaluated with inconsistency index $\left(\mathrm{I}^{2}\right)$ tests and was classified as moderate $\left(\mathrm{I}^{2} \geq 30 \%\right)$, substantial $\left(I^{2} \geq 50 \%\right)$ or considerable $\left(I^{2} \geq 75 \%\right)$. If heterogeneity existed, a random effects model was used to perform the meta-analysis. For sensitivity and specificity, the Spearman correlation coefficient between those two parameters was calculated to evaluate a threshold effect to determine heterogeneity. Sensitivity analysis was performed by recalculating the summary statistics after removing a single study or groups of studies from the analysis based on characteristics of the study design and aetiology.

\section{Patient and public involvement}

This study did not include patient or public involvement.

\section{RESULTS}

Literature search

Our literature search process is demonstrated in figure 1. Of the 210 studies identified through the database search, 108 were excluded after removing duplicates. Of the remaining 102 articles screened for eligibility, 87 were excluded after the review of the titles and abstracts, of which 48 were not in the field of interest while the rest were not clinical trials or observational studies. Full texts of the remaining 15 articles were retrieved with nine

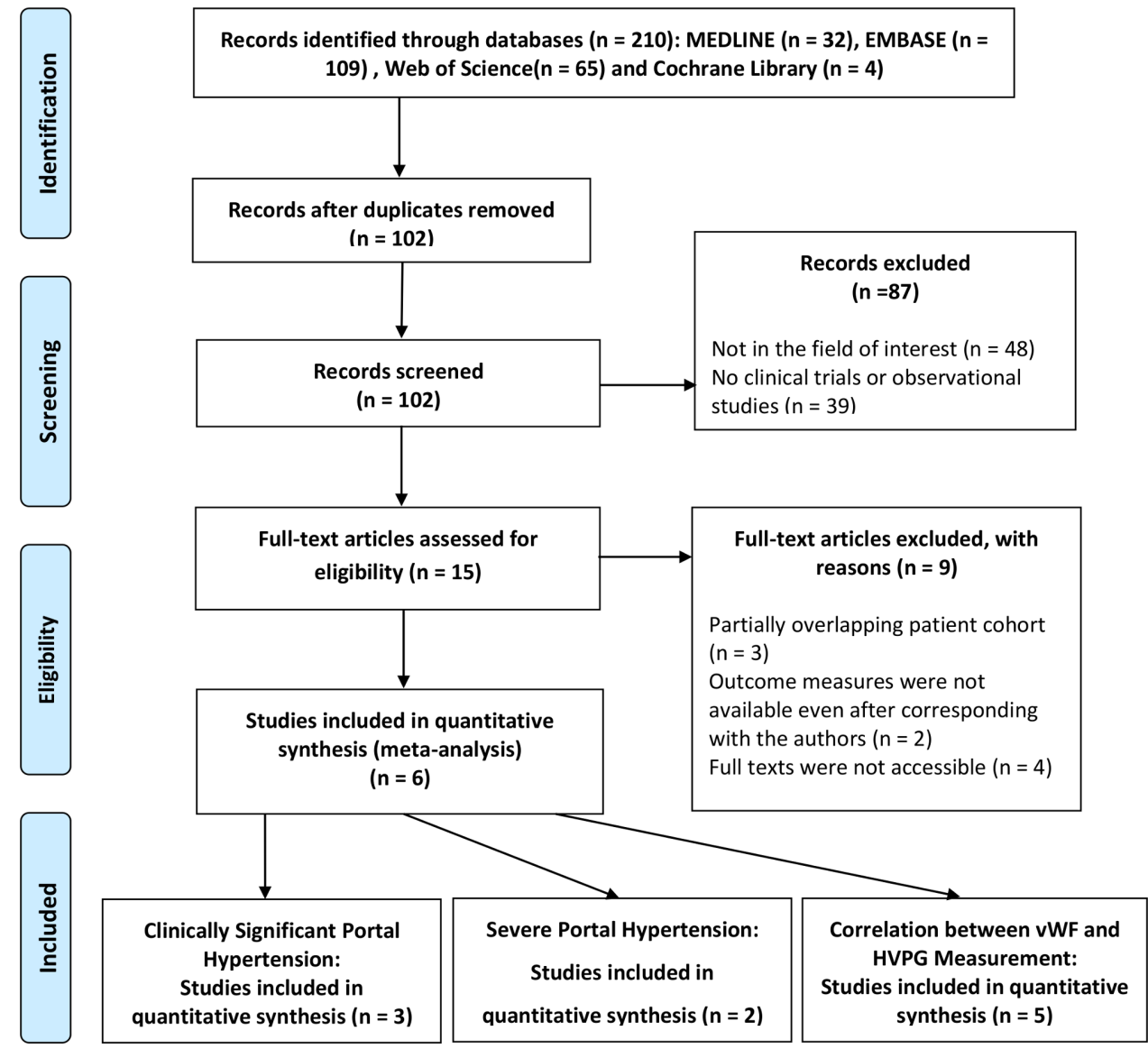

Figure 1 Flow diagram of study selection process. HVPG, hepatic venous pressure gradient; vWF, von Willebrand factor. 
articles excluded: three articles with partially overlapping patient cohorts; the outcome of two articles was not available even after corresponding with the authors; and the full text was inaccessible for four studies.

Thus, the remaining six studies were used for qualitative and quantitative review. ${ }^{711} 17-19$

\section{Quality assessment}

The result of the quality assessment is shown in online supplementary figure 1 . The median quality (QUADAS) score ranged from 12 to 14 . According to the results, two-thirds of the studies had a low risk of bias in patient selection; the main reason is that these four articles were cohort studies, while the remaining articles with a high risk of bias were case-control studies. Inappropriate exclusion may be the main reason for significant patient selection bias. A case-control study is retrospective; it begins with an outcome to comprehend the cause. However, cohort research studies a group of people who share similar characteristics for a long period of time, which is prospective. Thus, researchers in retrospective studies tend to choose patients who have more positive effects on their study results.

\section{Study characteristics}

A total of 994 patients were included in this meta-analysis. The basic characteristics of the final six studies are summarised in table 1 . All of the included studies were published after 2010. Furthermore, 67\% (4/6) of the studies were prospective in design versus retrospective $(33 \%, 2 / 6)$. The patient aetiology in the included studies was composed of a number of different diseases. The study population of people with alcoholic liver disease $(n=282)$ was involved in four studies, viral hepatitis $(n=260)$ in five studies and the aetiology of the remaining patients was unclear. The diagnostic criteria of PH among the six included studies were all HVPG. Regarding the outcome of interest, three studies investigated the diagnostic accuracy in diagnosing CSPH, and two studies investigated the accuracy in diagnosing SPH. In addition, five out of the six studies investigated the correlation between vWF and HVPG measurement. The median follow-up (months) of patients varied among the studies and ranged from 12 to 33 months.

\section{Meta-analysis for the correlation coefficient between vWF and HVPG measurement}

The forest plot of the correlation analysis between vWF and HVPG, which involved five studies, is presented in figure 2. The $\mathrm{I}^{2}$ statistic was $89 \%$, indicating significant heterogeneity between the studies. Based on the random effects model, the pooled correlation coefficient was 0.54 (95\% CI 0.35 to 0.69 ), suggesting a fairly moderate correlation between vWF and HVPG using the angiointerventional method with the $\mathrm{mm} \mathrm{Hg}$ scale.

Sensitivity analysis was performed for correlation coefficient analysis (online supplementary table 1). Studies were divided into groups according to study design (prospective, retrospective) and population aetiology (virus-associated cirrhosis). The sensitivity analysis failed to reveal a source for the heterogeneity. Nevertheless, no publication bias existed among the studies (online supplementary figure 2).

\section{Meta-analysis for diagnosing accuracy}

For $\mathrm{vWF}$ as a measurement to diagnose CSPH, three studies had sufficient data to calculate sensitivity and specificity. In these studies, the descriptive statistics for the diagnostic accuracy of vWF to diagnose CSPH, including sensitivity, specificity, PLR, NLR and DOR, are presented in table 2 . The pooled sensitivity and specificity were $82 \%$ (95\% CI 78 to 86 ) and $76 \%$ (95\% CI 68 to 83 ), respectively (figure 3$)$. The summary PLR was $3.11(95 \%$ CI 1.99 to 4.86 ), and NLR was 0.21 (95\% CI 0.11 to 0.40 ) (online supplementary figure 3 ). The AUC was $0.87(95 \%$ CI 0.80 to 0.94 ) (figure 4 ), and the pooled DOR was 15.50 (95\% CI 6.30 to 38.15 ) (online supplementary figure 4). No publication bias existed among these studies in the funnel plot since the Egger's test result was non-significant $(\mathrm{p}=0.848)$, and the shape of the funnel plot was symmetric (online supplementary figure 5).

Additionally, regarding vWF as a measurement to diagnose SPH, two studies had sufficient data to calculate sensitivity and specificity. In these studies, the descriptive statistics for the diagnostic accuracy of vWF for diagnosing SPH, including sensitivity, specificity, PLR, NLR and DOR, are presented in table 2 . The pooled sensitivity and specificity were $86 \%$ (95\% CI 80 to 90 ) and $75 \%$ (95\% CI 66 to 83 ), respectively (figure 3 ). The summary PLR was 3.43 (95\% CI 2.49 to 4.72 ), and the summary NLR was 0.19 (95\% CI 0.14 to 0.27 ) (online supplementary figure $6)$, while the pooled DOR was 17.91 (95\% CI 10.24 to 31.33) (online supplementary figure 7). As a result of less than three studies being involved, the SROC curve and the publication bias could not be calculated.

\section{DISCUSSION}

Diagnosing CSPH and SPH without the use of invasive strategies such as HVPG can be quite challenging. ${ }^{6} \mathrm{~A}$ delay in diagnosis can lead to significant morbidity and mortality. vWF is a non-invasive tool that can be used for diagnosis and prognosis. ${ }^{78}$ Our study is the first meta-analysis that summarises the correlation between vWF and HVPG as measurements to diagnose $\mathrm{PH}$ and the diagnostic accuracy of vWF to detect CSPH and SPH.

We included six studies of 994 patients in total, with the majority of studies being prospective in design. Our study revealed that $\mathrm{vWF}$ was moderately correlated with HVPG for $\mathrm{PH}$ detection. As evidenced by the pooled sensitivity and specificity of $82 \%$ and $76 \%$, respectively, and an AUC of 0.87 , the diagnostic accuracy of vWF to diagnose CSPH was satisfactory. These results indicate that vWF may be a reliable clinical index without the need of invasive procedures to assess $\mathrm{PH}$ independently and can be used for the diagnosis of CSPH. A universal theory to explain the 


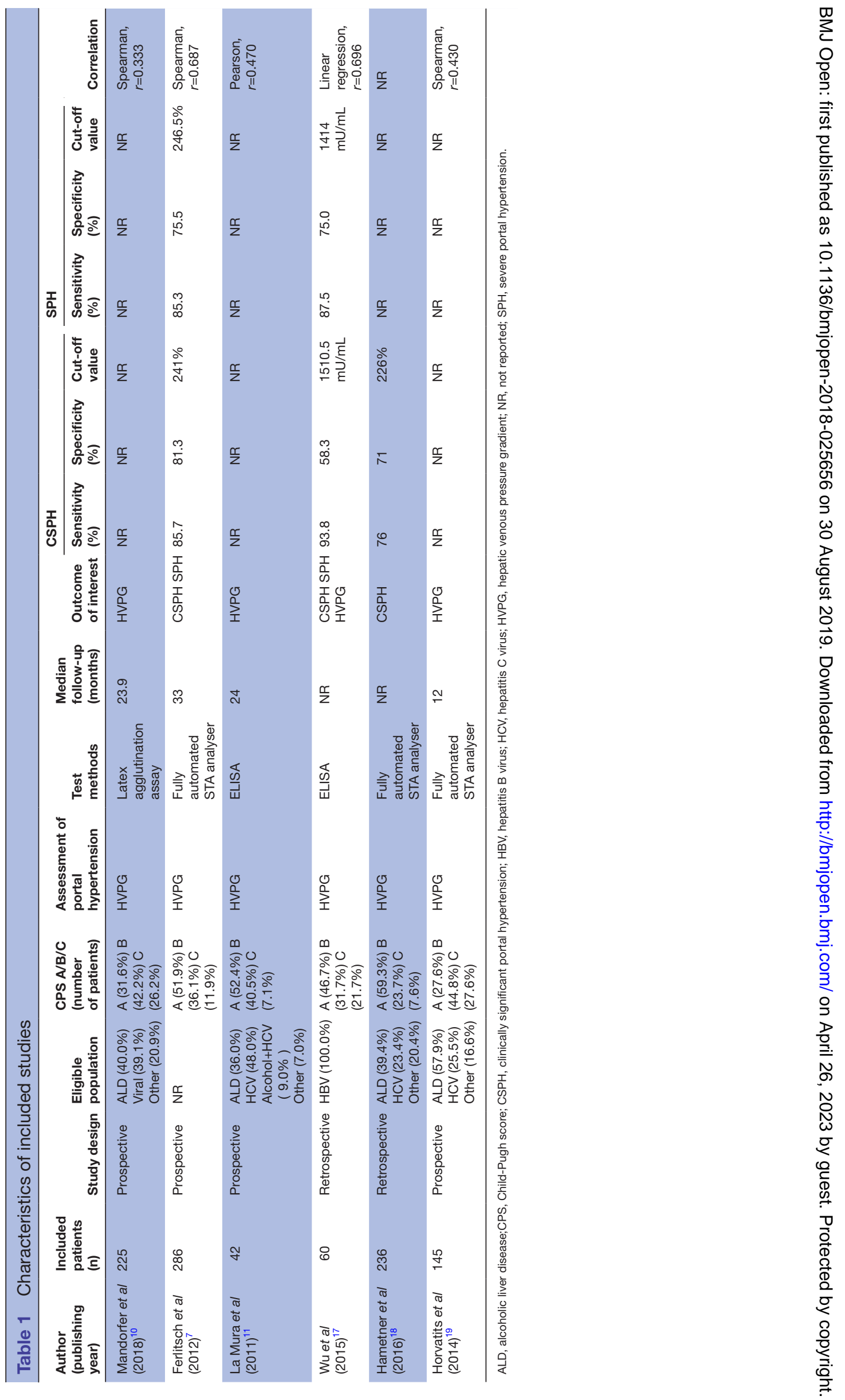




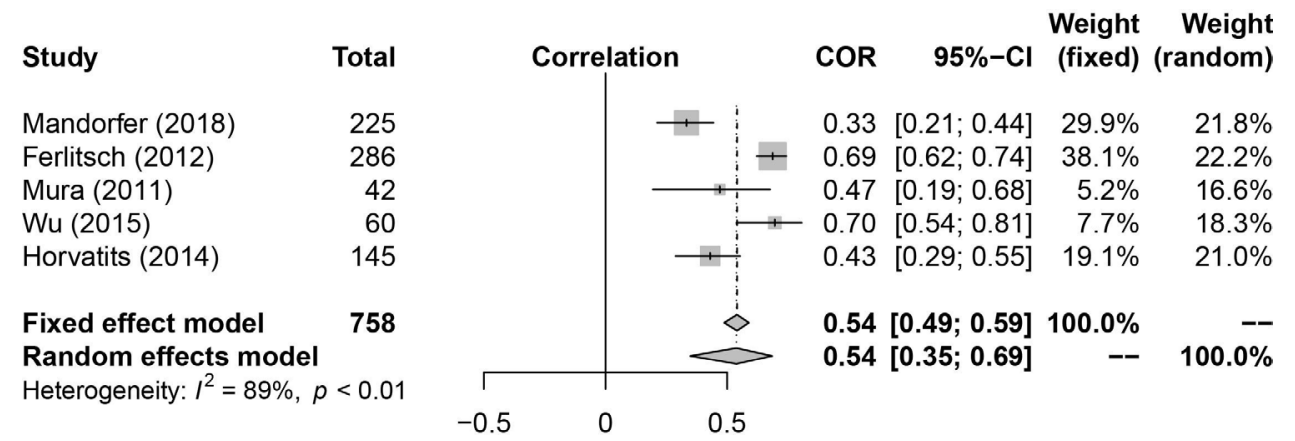

Figure 2 Forest plots of the correlation coefficient between von Willebrand factor (vWF) and hepatic venous pressure gradient (HVPG.

physiopathological mechanism of vWF in patients with cirrhosis was beyond the scope of our study. The elevated levels of $\mathrm{VWF}$ in cirrhosis might be a consequence of activated endothelial cells responsible for the interaction between the subendothelium at sites of vascular injury and platelets or endothelial instability resulting from increased shear stress, bacterial infection ${ }^{20}$ or even the induced expression of vWF in the cirrhotic liver itself. ${ }^{21}$ A reduced clearance of $\mathrm{vWF}$ results from the decreased expression or activity of ADAMTS13 (vWF-Ag cleaving protease). Decreased ADAMTS13 activity is related to capillary occlusion in thrombotic microangiopathy. ${ }^{22}$ If variation in these factors occurs in the liver, this would potentially influence the natural history of liver disease, gradually intensify $\mathrm{PH}$ and therefore underlie their predictive value and diagnostic significance. The fact that vWF mRNA has been shown to be an enhanced factor in the liver endothelium of patients with cirrhosis suggests that the liver endothelium may be a source of increased levels of vWF in patients with cirrhosis, consistent with the well-demonstrated hepatic endothelial dysfunction in cirrhosis. ${ }^{23}{ }^{24}$ Regarding the relatively low correlation coefficient in the previous study, ${ }^{10}$ vWF predicted the development of variceal bleeding (9\% increase in risk per $10 \% \mathrm{vWF}$ ) independently of well-established risk factors (history of variceal bleeding and the value of HVPG). This indicated that endothelial dysfunction might be a potential mechanism of variceal bleeding even beyond HVPG measurement. ${ }^{25}$

Other novel diagnostic measurements also showed satisfactory diagnostic performance for CSPH detection (ie, TE). ${ }^{9}$ Liver cirrhosis measured by TE was also highly correlated with HVPG, as evidenced by the summary sensitivity and specificity of $87.5 \%$ and $85.3 \%$, respectively. A higher TE value (ie, $\geq 20-25 \mathrm{kPa}$ ) alone or combined platelet count and spleen size could diagnose CSPH non-invasively according to a previous study, ${ }^{26}$ which suggested that TE would be more appropriate for screening and monitoring rather than diagnosing. In addition, previous studies assessed interleukin 6 and lipopolysaccharide (LPS)-binding protein, which is an acute phase protein synthesised in response to circulating endotoxins/LPS. ${ }^{27}$ Furthermore, C-reactive protein has also been shown to be of great prognostic value in patients with cirrhosis, ${ }^{28-30}$ even in the absence of active bacterial infections. The level of vWF showed an advantage over other novel measurements with its cost of US\$6 in standard laboratories per patient. ${ }^{7}$ However, certain known limitations of the application of vWF levels in patients with cirrhosis must be considered because it could be influenced by various factors, such as infections, malignancies, physical training or interferon therapy, which have also been shown to elevate vWF levels. ${ }^{31}$

In our meta-analysis, the most critical issue in the calculation of the summary estimates of the diagnostic accuracy was the between-study heterogeneity. As presented in table 1, various cut-off values were adopted among the six included studies. In general, higher cut-off values accounted for lower sensitivity and higher specificity. The cut-off value to diagnose CSPH adopted in different studies varied greatly. However, the computed diagnostic threshold showed a negative outcome with $\mathrm{p}=0.667$. As a result of less than three studies being included in the diagnosis of SPH, we did not analyse the heterogeneity

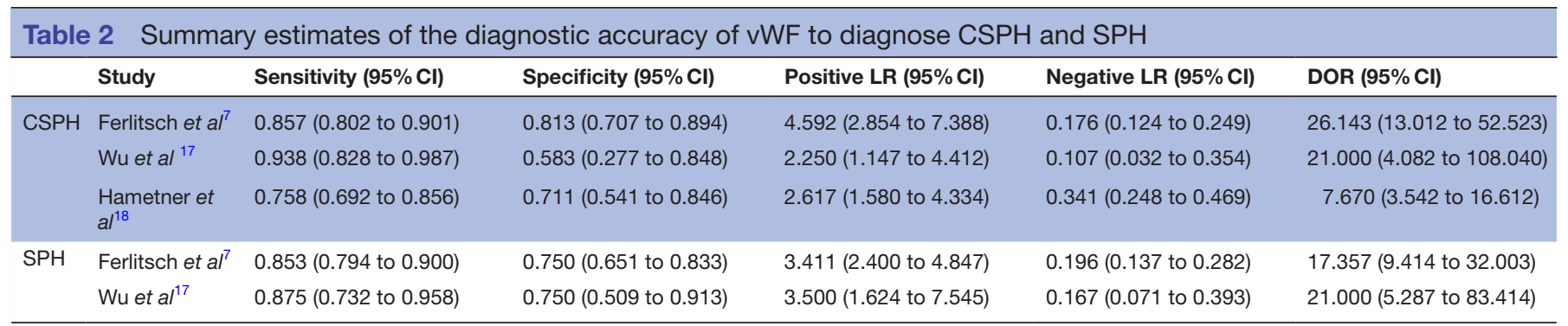

$\mathrm{CSPH}$, clinically significant portal hypertension; DOR, diagnostic OR;LR, likelihood ratio; SPH, severe portal hypertension; vWF, von Willebrand factor. 

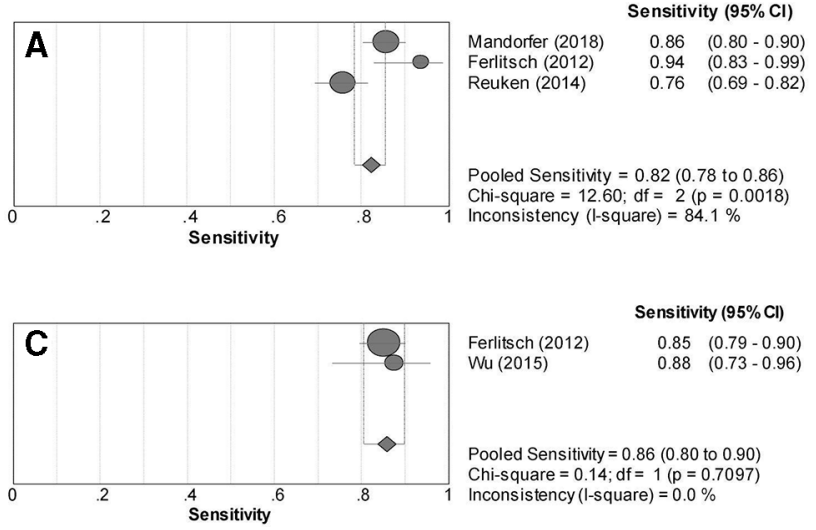

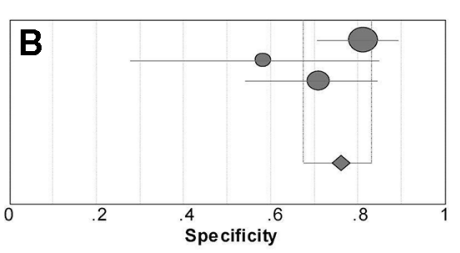

Specificity $(95 \% \mathrm{Cl})$

Mandorfer (2018) $\quad 0.81 \quad(0.71-0.89)$ \begin{tabular}{lll} 
Ferlitsch (2012) & 0.58 & $(0.28-0.85)$ \\
\hline & 0.71 & $(0.54-0.85)$
\end{tabular} Reuken (2014)

Pooled Specificity $=0.76(0.68$ to 0.83$)$ Chi-square $=3.54 ; \mathrm{df}=2(\mathrm{p}=0.1704)$ Inconsistency (I-square) $=43.5 \%$

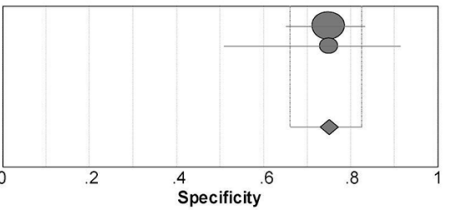

Ferlitsch (2012)

Specificity $(95 \% \mathrm{Cl})$

$0.75(0.65-0.83)$ Wu $0.75(0.65-0.83)$

Pooled Specificity $=0.75(0.66$ to 0.83$)$ Chi-square $=0.00 ; \mathrm{df}=1(\mathrm{p}=1.0000)$ Inconsistency (i-square) $=0.0 \%$

Figure 3 Forest plots of pooled sensitivity (A) and specificity (B) for von Willebrand factor (vWF) as markers of clinically significant portal hypertension (CSPH) and forest plots of pooled sensitivity (C) and specificity (D) for vWF as markers of severe portal hypertension (SPH).

source of SPH diagnosis. In the sensitivity analysis of the correlation coefficient (online supplementary table 1), the $\mathrm{I}^{2}$ statistic of patients with virus-associated $\mathrm{PH}$ was reduced from $91 \%$ to $65 \%(<75 \%)$, suggesting that the inhomogeneity in aetiology of the included studies may be the source of heterogeneity. Further studies investigating the diagnostic accuracy of vWF with different aetiologies are expected.

The strengths of our study include its well-validated systematic review methods, the reliable calculation procedure and the results reported based on the PRISMA guidelines. The advantage of this meta-analysis over other studies includes the following:
1. For our meta-analysis, a comprehensive search in multiple databases and a standardised assessment of methodological quality were conducted with a sufficient assessment of heterogeneity.

2. All the included studies were published before 5 April 2018, suggesting that our meta-analysis was an update of previous meta-analyses.

3. Our main research targets were the diagnostic correlation between vWF and HVPG and the diagnostic accuracy of CSPH and SPH in patients with liver cirrhosis. A similar meta-analysis ${ }^{32}$ discussed the biochemical mechanism and predictive effect of vWF in diagnosing patients with liver cirrhosis without the same classifica-

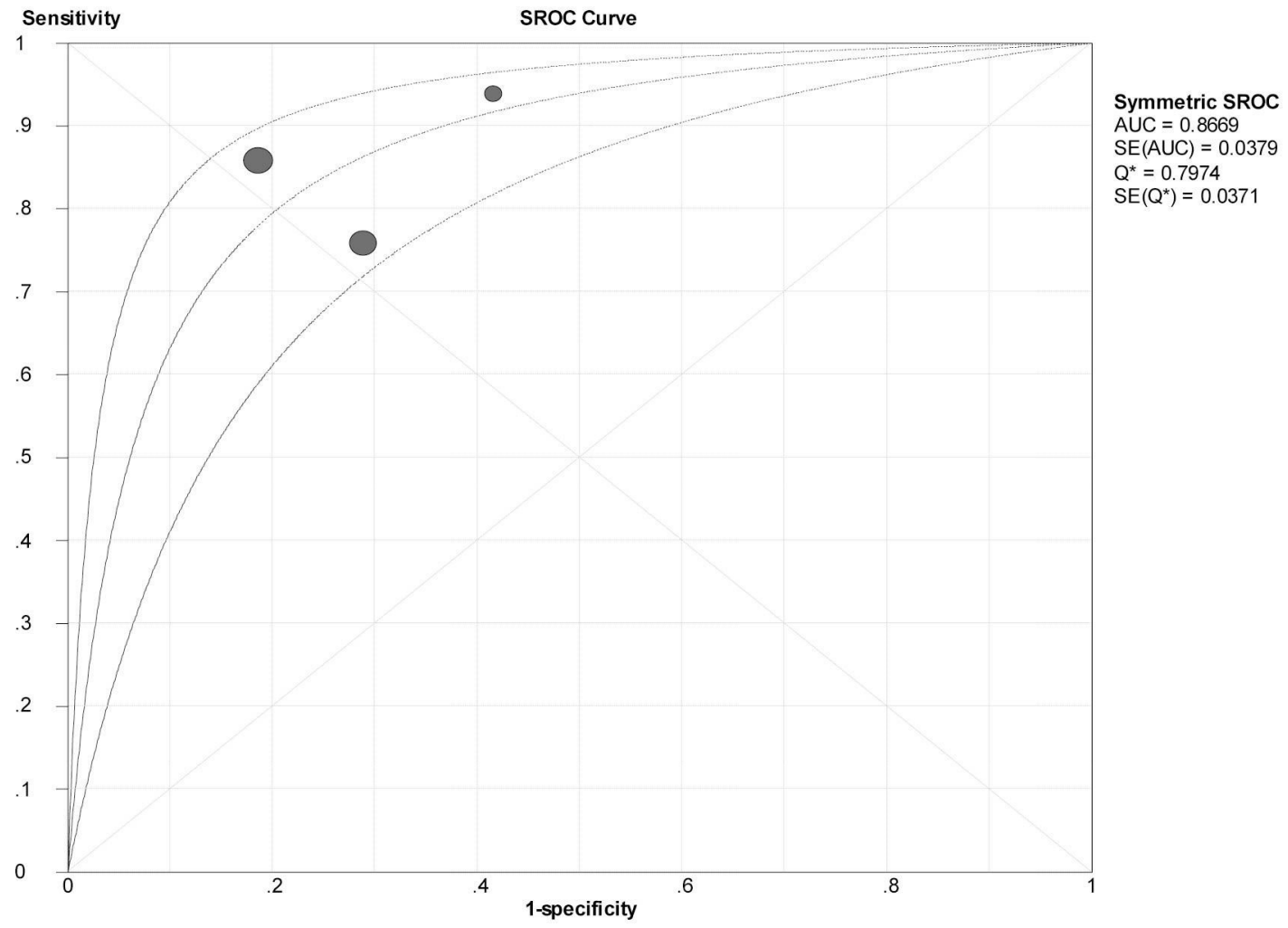

Figure 4 Summary receiver operating characteristic (SROC) curves of von Willebrand factor (vWF) as markers of clinically significant portal hypertension (CSPH). AUC, area under the receiver operating characteristic curve. 
tion, which was defined as CSPH as HVPG $\geq 10 \mathrm{~mm} \mathrm{Hg}$ and $\mathrm{SPH}$ as HVPG $\geq 12 \mathrm{~mm} \mathrm{Hg}$. Our research focused on this category and calculated the sensitivity and specificity separately.

4. PH was evaluated by the measurement of HVPG in the included studies according to international standards, as described previously. ${ }^{33} 34$

5. A sensitivity analysis and threshold effects were performed to explore the source of heterogeneity.

Our study has several limitations as follows:

1. Our research included a relatively small number of studies (six studies), of which two were retrospective with low-level evidence.

2. The eligible population of the included studies differed slightly in its aetiology.

3. A clear source of between-study heterogeneity was not found in this meta-analysis.

4. There were restrictions in the test method and cut-offs owing to the relatively small number of experimental data.

\section{CONCLUSIONS}

Consequently, available data suggested that $\mathrm{vWF}$ as a biomarker for the diagnosis of CSPH and SPH in patients with liver cirrhosis moderately correlated with the HVPG measurement. Considering the advantage of vWF, such as its non-invasiveness, low cost and relatively high diagnostic accuracy, vWF may be an effective diagnostic tool for CSPH and SPH.

\section{Author affiliations}

${ }^{1}$ CHESS Center, Institute of Portal Hypertension, The First Hospital of Lanzhou University, Lanzhou, China

${ }^{2}$ The First School of Clinical Medicine, Nanfang Hospital, Southern Medical University, Guangzhou, China

${ }^{3}$ Guangdong Traditional Medical and Sports Injury Rehabilitation Research Institute, Guangdong Second Provincial General Hospital, Guangzhou, China

${ }^{4}$ Second Department of General Surgery, Guangdong Second Provincial General Hospital, Guangzhou, China

${ }^{5}$ Department of Medical Imaging, Guangdong Second Provincial General Hospital, Guangzhou, China

${ }^{6}$ Department of Gastroenterology, Shandong Provincial Hospital Affiliated to Shandong University, Jinan, China

${ }^{7}$ Department of Radiology, Zhongda Hospital, Medical School of Southeast University, Nanjing, China

Author contributions Study concepts and design: XQ, ZZ, JT. Literature search: ZZ, XY. Data extraction: ZZ, CL, XL, XM. Data analysis: ZZ, CZ, SJ. Manuscript preparation: XY. Critical revision: XQ. All authors have participated sufficiently in the study and approved the final version.

Funding This work was supported by grants from the National Natural Science Foundation of China (81600510); Guangdong Science Fund for Distinguished Young Scholars (2018B030306019); Guangzhou Industry-Academia-Research Collaborative Innovation Major Project (201704020015); the President Foundation of Nanfang Hospital, Southern Medical University (2017Z012).

Competing interests None declared.

Patient consent for publication Not obtained.

Provenance and peer review Not commissioned; externally peer reviewed.

Data availability statement Data are available in a public, open access repository. All data relevant to the study are included in the article or uploaded as supplementary information.
Open access This is an open access article distributed in accordance with the Creative Commons Attribution Non Commercial (CC BY-NC 4.0) license, which permits others to distribute, remix, adapt, build upon this work non-commercially, and license their derivative works on different terms, provided the original work is properly cited, appropriate credit is given, any changes made indicated, and the use is non-commercial. See: http://creativecommons.org/licenses/by-nc/4.0/.

\section{REFERENCES}

1. Blachier M, Leleu H, Peck-Radosavljevic M, et al. The burden of liver disease in Europe: a review of available epidemiological data. $J$ Hepatol 2013;58:593-608.

2. Ripoll C, Groszmann R, Garcia-Tsao G, et al. Hepatic venous pressure gradient predicts clinical decompensation in patients with compensated cirrhosis. Gastroenterology 2007;133:481-8.

3. Reiberger T, Mandorfer M. Beta adrenergic blockade and decompensated cirrhosis. J Hepatol 2017;66:849-59.

4. Abraldes JG, Bureau C, Stefanescu H, et al. Noninvasive tools and risk of clinically significant portal hypertension and varices in compensated cirrhosis: The "Anticipate" study. Hepatology 2016;64:2173-84.

5. D'Amico G, Pasta L, Morabito A, et al. Competing risks and prognostic stages of cirrhosis: a 25-year inception cohort study of 494 patients. Aliment Pharmacol Ther 2014;39:1180-93.

6. de Franchis R, Baveno V Faculty. Revising consensus in portal hypertension: report of the Baveno V consensus workshop on methodology of diagnosis and therapy in portal hypertension. $J$ Hepatol 2010;53:762-8.

7. Ferlitsch M, Reiberger T, Hoke M, et al. Von Willebrand factor as new noninvasive predictor of portal hypertension, decompensation and mortality in patients with liver cirrhosis. Hepatology 2012;56:1439-47.

8. Yildiz M, Sahin A, Behnes M, et al. An expanding role of biomarkers in pulmonary arterial hypertension. Curr Pharm Biotechnol 2017;18:491-4

9. You M-W, Kim KW, Pyo J, et al. A meta-analysis for the diagnostic performance of transient elastography for clinically significant portal hypertension. Ultrasound Med Biol 2017;43:59-68.

10. Mandorfer M, Schwabl P, Paternostro R, et al. Von Willebrand factor indicates bacterial translocation, inflammation, and procoagulant imbalance and predicts complications independently of portal hypertension severity. Aliment Pharmacol Ther 2018;47:980-8.

11. La Mura V, Reverter JC, Flores-Arroyo A, et al. Von Willebrand factor levels predict clinical outcome in patients with cirrhosis and portal hypertension. Gut 2011;60:1133-8.

12. Maieron A, Salzl P, Peck-Radosavljevic M, et al. Von Willebrand factor as a new marker for non-invasive assessment of liver fibrosis and cirrhosis in patients with chronic hepatitis C. Aliment Pharmacol Ther 2014;39:331-8.

13. Kalambokis GN, Oikonomou A, Christou L, et al. Von Willebrand factor and procoagulant imbalance predict outcome in patients with cirrhosis and thrombocytopenia. J Hepatol 2016;65:921-8.

14. Page MJ, Moher D. Evaluations of the uptake and impact of the preferred reporting items for systematic reviews and meta-analyses (PRISMA) statement and extensions: a scoping review. Syst Rev 2017;6:263

15. Whiting PF, Rutjes AWS, Westwood ME, et al. QUADAS-2: a revised tool for the quality assessment of diagnostic accuracy studies. Ann Intern Med 2011;155:529-36.

16. Whiting P, Rutjes AWS, Reitsma JB, et al. The development of QUADAS: a tool for the quality assessment of studies of diagnostic accuracy included in systematic reviews. BMC Med Res Methodol 2003;3:25.

17. Wu H, Yan S, Wang G, et al. Von Willebrand factor as a novel noninvasive predictor of portal hypertension and esophageal varices in hepatitis B patients with cirrhosis. Scand J Gastroenterol 2015;50:1160-9.

18. Hametner S, Ferlitsch A, Ferlitsch M, et al. The vitro score (von Willebrand factor Antigen/Thrombocyte ratio) as a new marker for clinically significant portal hypertension in comparison to other non-invasive parameters of fibrosis including ELF test. PLOS One 2106;11:e0149230.

19. Horvatits T, Drolz A, Roedl K, et al. Von Willebrand factor antigen for detection of hepatopulmonary syndrome in patients with cirrhosis. $J$ Hepatol 2014;61:544-9.

20. Ferro D, Quintarelli C, Lattuada A, et al. High plasma levels of von Willebrand factor as a marker of endothelial perturbation in cirrhosis: relationship to endotoxemia. Hepatology 1996;23:1377-83. 
21. Hollestelle MJ, Geertzen HGM, Straatsburg IH, et al. Factor VIII expression in liver disease. Thromb Haemost 2004;91:267-75.

22. Moake JL. Thrombotic microangiopathies. N Engl J Med 2002;347:589-600.

23. Iwakiri Y, Groszmann RJ. Vascular endothelial dysfunction in cirrhosis. J Hepatol 2007;46:927-34.

24. Matei V, Rodríguez-Vilarrupla A, Deulofeu R, et al. The eNOS cofactor tetrahydrobiopterin improves endothelial dysfunction in livers of rats with CCl4 cirrhosis. Hepatology 2006;44:44-52.

25. Bellot P, García-Pagán JC, Francés R, et al. Bacterial DNA translocation is associated with systemic circulatory abnormalities and intrahepatic endothelial dysfunction in patients with cirrhosis. Hepatology 2010;52:2044-52.

26. de Franchis R, Baveno VI Faculty . Expanding consensus in portal hypertension: report of the Baveno $\mathrm{VI}$ consensus workshop: Stratifying risk and individualizing care for portal hypertension. $J$ Hepatol 2015;63:743-52.

27. Reiberger T, Ferlitsch A, Payer BA, et al. Non-Selective betablocke therapy decreases intestinal permeability and serum levels of LBP and IL-6 in patients with cirrhosis. $J$ Hepatol 2013;58:911-21.

28. Cervoni J-P, Thévenot $\mathrm{T}$, Weil $\mathrm{D}$, et al. C-Reactive protein predicts short-term mortality in patients with cirrhosis. $J$ Hepatol 2012;56:1299-304.
29. Di Martino V, Coutris C, Cervoni J-P, et al. Prognostic value of C-reactive protein levels in patients with cirrhosis. Liver Transp/ 2015;21:753-60.

30. Cervoni J-P, Amorós Àlex, Bañares R, et al. Prognostic value of C-reactive protein in cirrhosis: external validation from the CANONIC cohort. Eur J Gastroenterol Hepatol 2016;28:1028-34.

31. Homoncik M, Ferlitsch A, Ferenci P, et al. Short- and long-term effects of therapy with interferon-alpha and PEGylated interferonalpha/ribavirin on platelet plug formation and von Willebrand factor release in patients with chronic hepatitis C. Aliment Pharmacol Ther 2005;21:49-55.

32. Eapen CE, Elias JE, Mackie I, et al. Prognostic significance of von Willebrand factor in cirrhosis: a possible mechanism. Hepatology 2013;58.

33. Ferlitsch A, Teml A, Reinisch W, et al. 6-Thioguanine associated nodular regenerative hyperplasia in patients with inflammatory bowel disease may induce portal hypertension. Am J Gastroenterol 2007;102:2495-503.

34. Reiberger T, Rutter K, Ferlitsch A, et al. Portal pressure predicts outcome and safety of antiviral therapy in cirrhotic patients with hepatitis C virus infection. Clin Gastroenterol Hepatol 2011;9:602-8 